\title{
The beneficial vortex and best spatial arrangement in total extracardiac cavopulmonary connection
}

\author{
Antonio Amodeo, $\mathrm{MD}^{\mathrm{b}}$ \\ Mauro Grigioni, $\mathrm{MS}^{\mathrm{C}}$ \\ Guido Oppido, $M D^{\mathrm{b}}$ \\ Carla Daniele, $\mathrm{MS}^{\mathrm{c}}$ \\ Giuseppe D'Avenio, $\mathrm{MS}^{\mathrm{c}}$ \\ Giovanni Pedrizzetti, $\mathrm{PhD}^{\mathrm{d}}$ \\ Salvatore Giannico, MD ${ }^{\mathrm{a}}$ \\ Sergio Filippelli, $\mathrm{MD}^{\mathrm{b}}$ \\ Roberto M. Di Donato $M D^{b}$
} From the Department of Pediatric Cardiolo-
gy $^{\mathrm{a}}$ and Cardiac Surgery, ${ }^{\mathrm{b}}$ Bambino Gesù Hospital, and the Laboratory of Biomedical Engineering, Istituto Superiore di Sanità, ${ }^{\mathrm{c}}$ Rome, and the Faculty of Engineering, University of Trieste, Trieste, ${ }^{\text {, Italy. }}$

Received for publication May 5, 2001; revisions requested July 6, 2001; revisions received Aug 9, 2001; accepted for publication Sept 12, 2001.

Address for reprints: Antonio Amodeo, MD, Dipartimento Medico Chirurgico di Cardiologia Pediatrica, Ospedale Bambino Gesù, Piazza S. Onofrio 4, 00165, Rome, Italy (E-mail: antonioamodeo@yahoo.it).

J Thorac Cardiovasc Surg 2002;124:471-8

Copyright ( $(2) 2002$ by The American Association for Thoracic Surgery

0022-5223/2002 \$35.00+0 $\quad \mathbf{1 2 / 6 / 1 2 0 3 4 9}$

doi: $10.1067 / \mathrm{mtc} .2002 .120349$
Objective: Total extracardiac cavopulmonary connection is an established procedure, but the best spatial arrangement remains controversial. On the basis of our clinical experience with total extracardiac cavopulmonary connection, we performed quantitative and qualitative flow analysis on total extracardiac cavopulmonary connection models simulating the two most frequent arrangements applied to our patients to determine the most favorable hydrodynamic pattern.

Methods: We selected two main groups among 110 patients who underwent total extracardiac cavopulmonary connection, those with left-sided inferior vena cava anastomosis (type 1) and those with facing superior and inferior vena cava anastomoses (type 2). Blown-glass total extracardiac cavopulmonary connection phantom models were constructed on the basis of nuclear magnetic resonance and angiographic images. Flow measurements were performed with a Nd:YAG Q-switched laser and a particle imaging velocimetry system. A power dissipation study and a finite-element numeric simulation were also carried out.

Results: When applying superior and inferior vena caval flow proportions of total systemic venous return of $40 \%$ and $60 \%$, respectively, a vortex was visualized in the type 1 phantom that rotated counterclockwise at the junction of the caval streams. This apparent vortex was not a true vortex; rather, it represented a weakly dissipative recirculating zone modulating the flow distribution into the pulmonary arteries. The power dissipation and finite-element numeric stimulation confirmed the beneficial nature of the apparent vortex and a more energy-saving pattern in the type 1 phantom than in the type 2 phantom.

Conclusion: Total extracardiac cavopulmonary connection with left-sided diversion of the inferior vena caval conduit anastomosis is characterized by a central vortex that regulates the caval flow partitioning and provides a more favorable energysaving pattern than is seen with the total extracardiac cavopulmonary connection with directly opposed cavopulmonary anastomoses.

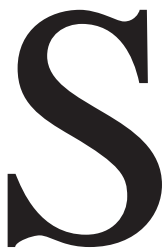

ince its first description in $1971,{ }^{1}$ the Fontan operation has undergone several modifications geared toward the goal of energy preservation to minimize systemic venous pressure and ventricular workload in the cavopulmonary system, which is assumed to enhance clinical outcome. ${ }^{2-5}$ In 1988, de Leval and colleagues ${ }^{2}$ introduced the concept of total cavopulmonary connection (TCPC), the combination of a bidirectional Glenn anastomosis with a tubular intracardiac inferior vena cava (IVC) 
extension to the pulmonary arteries, because of the notion that streamlined parallel flows ensure the most efficient hydrodynamic pattern.

In recent years, increasingly sophisticated in vitro, ${ }^{6-9}$ in vivo, ${ }^{10-14}$ and computational ${ }^{15,16}$ studies on the best fluid dynamics among different spatial arrangements of TCPC have concurred that the two cavopulmonary anastomoses should not be placed in a face-to-face configuration but rather should be deflected to opposite sides. ${ }^{7,8}$ However, controversy still exists with respect to the side and the proper geometric orientation.

In our practice we have endorsed the use of total extracardiac cavopulmonary connection (TECPC) $)^{4,17}$ and have generally preferred to position the IVC conduit to the left of the bidirectional Glenn anastomosis. In fact, we have found it more hazardous to position the IVC conduit to the right, because it then potentially impinges on the right lower lobar artery. This study is based on a 12-year experience with TECPC and deals with in vitro quantitative and qualitative analyses of the flow fields simulating the two most frequent geometric patterns used at our institution. TECPC is a spatially complex system that demands a high-performance method for the flow field analysis. We have used a novel application of velocity fields generated by particle imaging velocimetry (PIV) that provides a highly accurate flow mapping, both spatially and temporally. A finite-element numeric model was also built on the basis of our in vitro measurements, and results were compared with the PIV results.

\section{Material and methods}

From an examination of the angiographic and magnetic resonance imaging data of 110 patients operated on with a TECPC, the two most frequently used geometric configurations of the "caval cross" were determined. Two blown-glass phantom circuits were created to replicate these configurations: the type 1 phantom, in which the IVC conduit was placed to the left of the Glenn anastomosis with a 6-mm offset (defined as the horizontal distance between the caval midpoints), and the type 2 phantom, in which the IVC conduit was directly opposed to the Glenn anastomosis, with zero offset. In the former configuration the IVC conduit, which was inclined toward the left pulmonary artery (LPA), had a $22^{\circ}$ angle with respect to the vertical axis. The dimensions of the phantoms were as follows: both superior vena cava (SVC) and IVC were $11.5 \mathrm{~mm}$ in diameter, and both LPA and right pulmonary artery (RPA) were $8.6 \mathrm{~mm}$ in diameter. With respect to body size, our phantom circulations corresponded to the averages for our patients of $10 \mathrm{~kg}$ of body weight and $0.5 \mathrm{~m}^{2}$ of body surface area: the flow rate of $1 \mathrm{~L} / \mathrm{min}$ corresponded to an indexed flow at rest of $2.0 \mathrm{~L} \cdot \mathrm{min}^{-1} \cdot \mathrm{m}^{-2}$ and was the baseline flow rate for our energy dissipation study.

\section{Fluidic System}

The blown-glass TECPC was used a in steady-flow regimen, because the residual pulsatility present in the venous return plays a limited, if not negligible, role. The phantom circulations were inserted in a closed loop, in which the driving pressures were provided by the gravimetric effect. The flow could be controlled in each branch of the connection by regulating the pressure of the corresponding outlet or inlet with suitable positioning of the associated reservoir. The changes in "pulmonary" arterial load were achieved by narrowing the tubes connected to either phantom pulmonary arterial branch. To mimic the typical viscosity of blood, the circulating fluid was a water-glycerol solution with a dynamic viscosity of $3.6 \mathrm{cP}$. The isolation of the outflow section of the reservoir from the incoming fluid, as provided by the baffle arrangements, guaranteed that the height of the fluid column driving the flow was constant during the measurements, thereby ensuring a stationary flow. A contribution of the flow-generating system to the observed effects in the TECPC therefore could be excluded. Pressure measurements were performed with a piezometric catheter (Millar Instruments, Inc, Houston, Tex), and the output voltage of the electronic equipment associated with the catheter was averaged with a digital voltmeter and subsequently converted to millimeters of mercury. This was done for each point of a predefined set of measurements points inside the SVC, IVC, RPA, and LPA, at $20 \mathrm{~mm}$ from the center of the blown-glass cross. These measurements were repeated at total flow rates of $1,2,3$, and 4 $\mathrm{L} / \mathrm{min}$. The flow ratios to the RPA and LPA were varied from 30:70, to 50:50, to 70:30. The caval flow rates in the SVC and IVC were fixed at 40:60 and 50:50, respectively.

\section{Flow Visualization and Measurement}

A qualitative analysis of flow visualization was obtained by using a sheet of Ar-ion continuous-wave laser light and a Kodak Ektapro high-speed videographic system (Eastman Kodak Company, Rochester, NY). The flow was seeded with 80- $\mu \mathrm{m}$ Amberlite particles to record 100 frames at 50, 125, and 250 frames per second.

Quantitative flow measurements were performed by means of a Quantel Twin Nd:YAG Q-switched laser (Quantel, Les Ulis, France) and a PIV system (Dantec Measurements Technology, Skovlunde, Denmark). The flow was seeded with $10-\mu \mathrm{m}$ silveredglass particles before the image recordings. The plane with the laser sheet was the symmetry plane of the TCPC, the plane containing the axes of the connected vessels. The Visiflow program (Atomic Energy Agency, Harwell, United Kingdom) was used for quantitative analysis of the flow field. Further details on the PIV technique are reported in Appendix 1.

\section{Power Dissipation}

To characterize the energetic dissipation and compare the different geometries, we calculated the total power dissipation as follows ${ }^{1}$ :

$$
\begin{gathered}
\mathrm{W}_{\text {loss }}=\mathrm{W}_{\text {static }}+\mathrm{W}_{\text {dyn }} \\
\mathrm{W}_{\text {static }}=\mathrm{P}_{\mathrm{I}} \mathrm{Q}_{\mathrm{I}}+\mathrm{P}_{\mathrm{S}} \mathrm{Q}_{\mathrm{S}}-\mathrm{P}_{\mathrm{R}} \mathrm{Q}_{\mathrm{R}}-\mathrm{P}_{\mathrm{L}} \mathrm{P}_{\mathrm{L}} \\
\mathrm{W}_{\text {dyn }}=\frac{\rho}{2} \mathrm{~V}_{\mathrm{I}}^{2} \mathrm{Q}_{\mathrm{I}}+\frac{\rho}{2} \mathrm{~V}_{\mathrm{S}}^{2} \mathrm{Q}_{\mathrm{S}}-\frac{\rho}{2} \mathrm{~V}_{\mathrm{R}}^{2} \mathrm{Q}_{\mathrm{R}}-\frac{\rho}{2} \mathrm{~V}_{\mathrm{L}}^{2} \mathrm{Q}_{\mathrm{L}}
\end{gathered}
$$

where $W_{\text {static }}$ represents the contribution from the static pressures, $W_{d y n}$ represents the contribution from the dynamic pressures, $P$ and $Q$ represent pressure and flow rates, respectively, $V$ (which is equal to $\mathrm{Q} / \mathrm{A}$, where $A$ represents area) is the average velocity in 


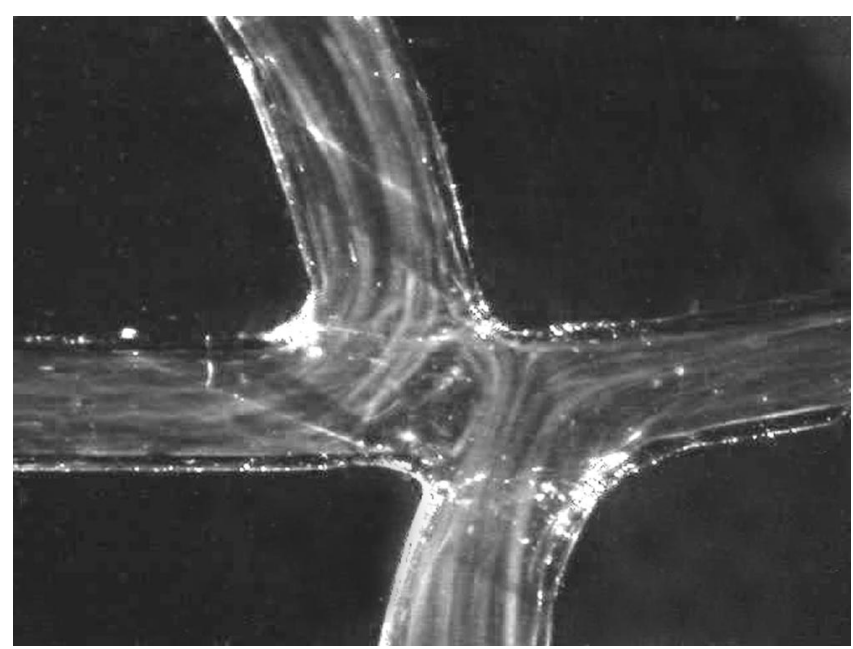

Figure 1. Flow visualization of type 1 phantom.

the considered section, and the subscripts $I, S, L$, and $R$ denote quantities relative to the IVC, SVC, LPA, and RPA, respectively. The control volume was the region between the inflow and the outflow sections placed at $20 \mathrm{~mm}$ from the center of the cross. The accurate specification of the extent of the region in which the power loss is calculated is important in the evaluation of the result.

\section{Finite-Element Numeric Simulation}

A finite-element numeric model was also built on the basis of our in vitro measurements, and the results were compared with the PIV data. This model was based on the vorticity stream function formulation of the 3-dimensional Navier-Stokes equations on the symmetry plane. The symmetry plane formulation represents an extension of the 2-dimensional approximation. Although 3-dimensional conservation laws are imposed in the symmetry plane, the model remains planar, and it is used in the first instance to guarantee a sufficient in-plane resolution in its finite-element application.

The use of about 1800 nodes, or 34,000 elements, was considered necessary to capture the details of the experimentally observed vortex structure. Such a formulation was applied to investigate the fluid dynamic behavior of TECPC geometry. Further details on the finite-element model application are reported in Appendix 2.

\section{Results}

\section{Flow Visualization}

In the type 1 phantom, when we applied a 40:60 SVC/IVC flow ratio, a balanced (equal) flow was obtained in the two pulmonary arteries, with preferential direction of the IVC flow to the LPA and of the SVC flow to the RPA. Streamlined, unidirectional flows toward the RPA from the SVC and toward the LPA from the IVC were noted. The flows remained streamlined, with a certain degree of symmetry, and generated a counterclockwise rotating central vortex as a consequence of the mean shearing forces generated by the two principal caval flows (Figure 1). This vortex appeared
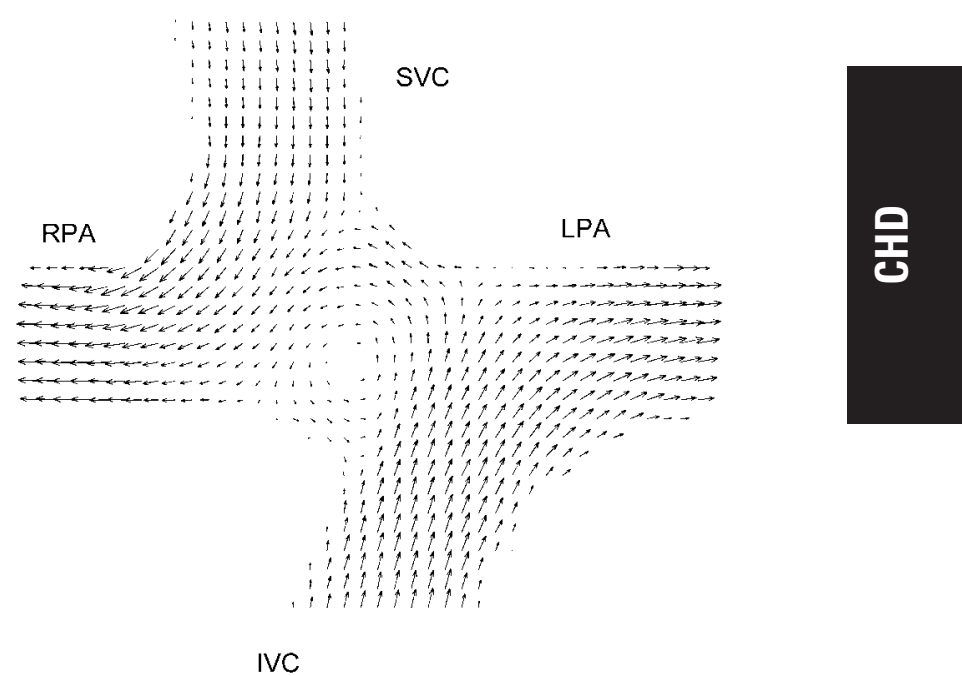

Figure 2. Velocity field relative to type 1 phantom, generated by PIV analysis, 40:60 SVC/IVC ratio.

to direct the inlet caval flows away from each other, avoiding significant collision but still allowing adequate mixing with a portion of the IVC flow diverted to the RPA, as shown by PIV analysis of flow field (Figure 2).

The numeric analysis of the flow field through the type 1 phantom confirmed the PIV analysis. Figure 3, $a$, reports the velocity streamlines and Figure 3, $b$, reports the vorticity field provided by the computational study, for a mean Reynolds number (Re) in the pulmonary arteries of 700 (corresponding to $1 \mathrm{~L} / \mathrm{min}$ in each pulmonary artery, or a total flow of $2 \mathrm{~L} / \mathrm{min}$ ). As shown in Figure 3, a, the computed streamlines exiting the IVC were mainly directed toward the LPA, but a portion of them turned to the other side of the cross, flowing tangential to the central structure. This effect can also be seen in the PIV velocity field of Figure 2. The flow characteristics in the SVC were well reproduced by the computational study. Thus the satisfactory simulation of the flow field by the numeric approach enabled exploitation of the higher spatial accuracy of the latter technique, particularly with respect to the flow near the vascular walls. The simulation confirmed that the apparent vortex was a weak, stagnating zone without vorticity at its center, surrounded by the vascular walls and by separated vorticity layers. It is evident from Figure 3, b, that the vorticity was low at the center of the cross, where the flow underwent a rotation; instead, the vorticity was higher in the boundary layer created at the pulmonary arterial level (on the left side of the SVC anastomosis) by the flow exiting from the IVC. These vorticity layers were thin and tend to be elongated toward the RPA. This behavior was even more evident for higher value of Re. In the central circulation zone depicted in Figure 3, $a$, the pressure does not show the drop at the center typical of a vortex. In this sense, the vortex could be defined 

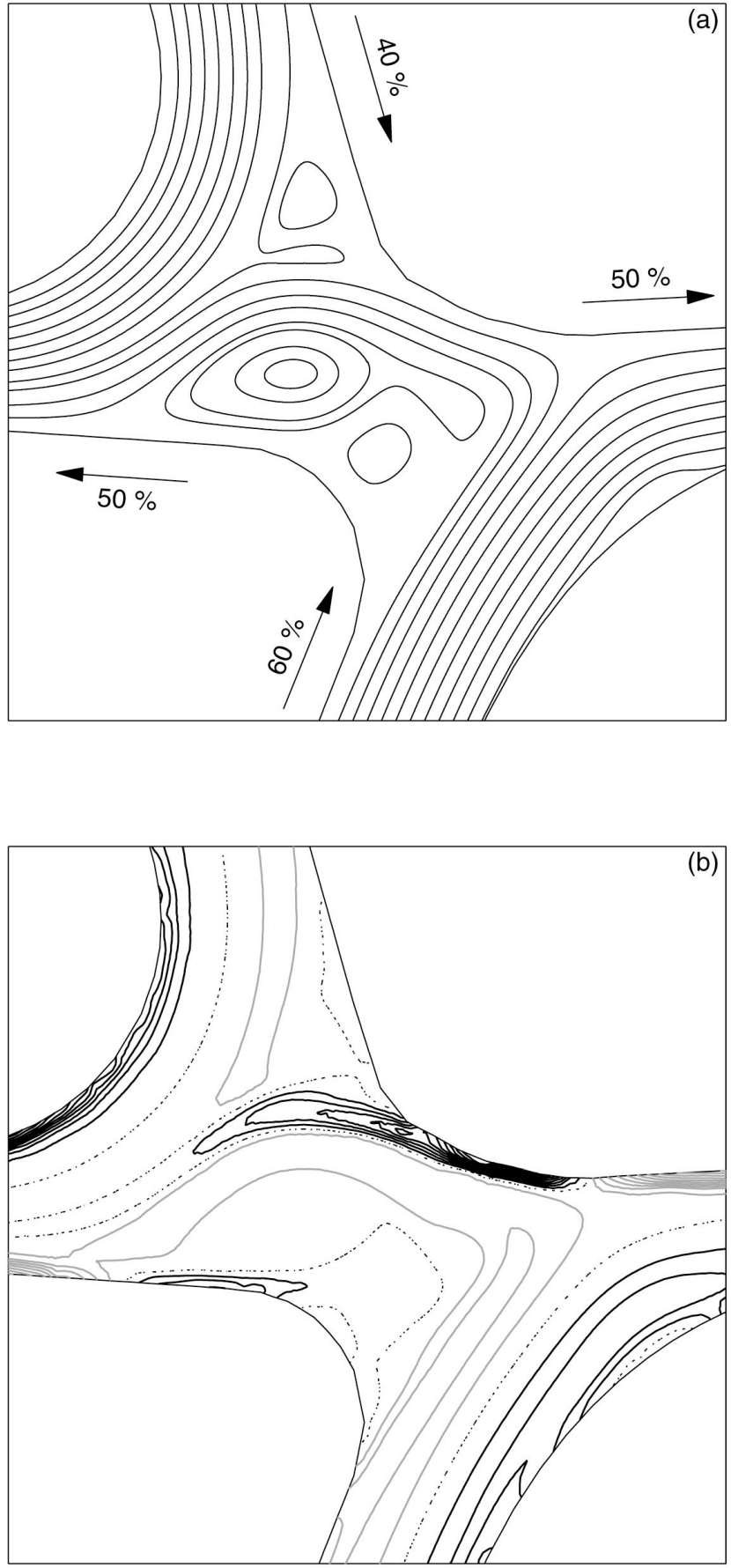

Figure 3. Numeric simulation of flow field in type 1 phantom corresponding to experimental case: Re 700 , IVC $60 \%$ of total venous return, SVC $40 \%$, equal pulmonary arterial flows. a, Streamlines. b, Vorticity fields contours, level from zero (dotted lines) with steps at \pm 0.5 . Gray lines represent positive levels; black lines represent negative levels.

as a weakly dissipative, beneficial flow division structure that helped regulate the flow partitioning of the caval flows toward either pulmonary artery.

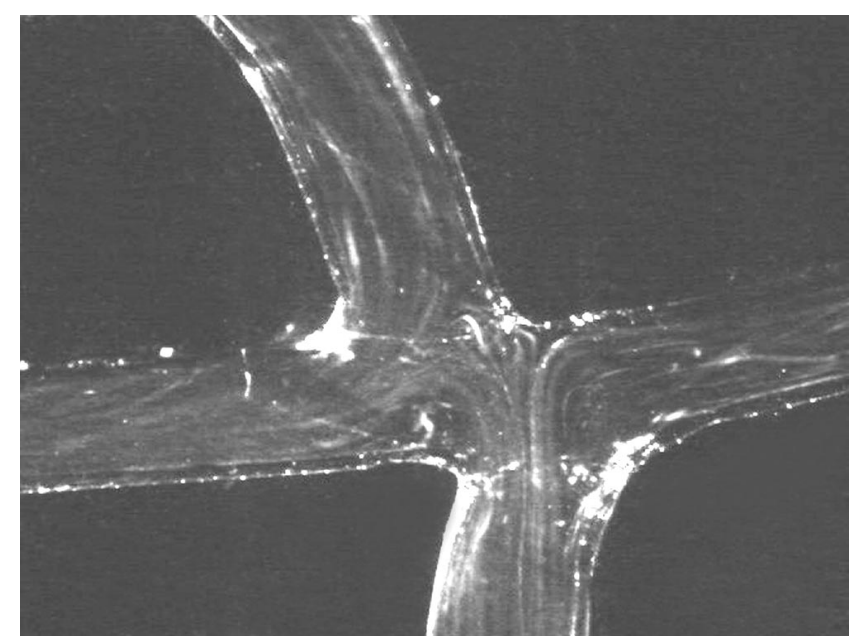

Figure 4. Flow visualization of type 1 phantom. LPA is stenosed, with RPA carrying $70 \%$ of pulmonary flow.

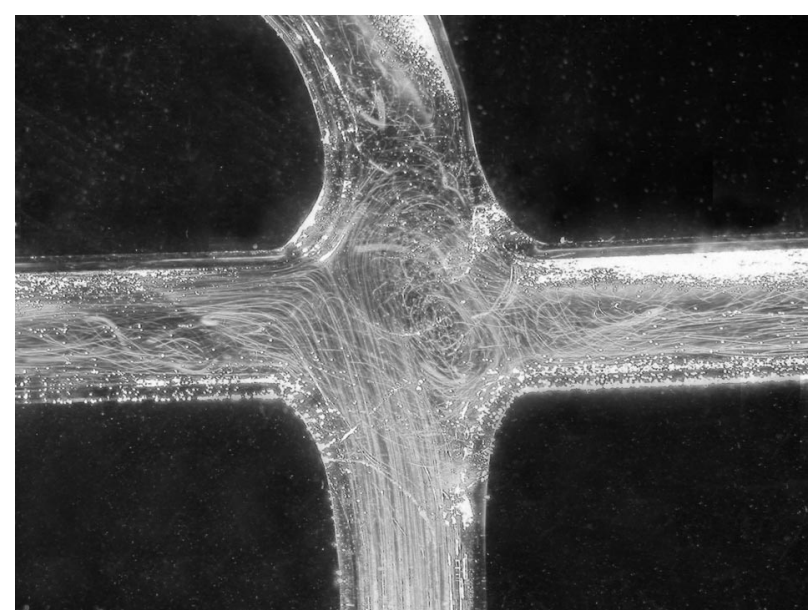

Figure 5. Flow visualization of type 2 phantom. Collision of converging caval flows generates circular swirling patterns.

When we applied a 50:50 SCV/IVC flow ratio, a slightly unbalanced pulmonary arterial flow was visualized, with the LPA carrying $57.5 \%$ of the total flow. The caval flows were still directed predominantly toward a preferential pulmonary artery (the IVC to the LPA and the SVC to the RPA). Pulmonary arterial flow distribution was slightly different than in the former case (Figure 2), with the vortex center shifted toward the LPA.

When we applied different RPA/LPA flow proportions, varying from 50:50 to 70:30 and 30:70, the position of the vortex shifted from the central position to the upper right or lower left part of the cross, away from the stenotic pulmonary artery, until it vanished (Figure 4). In this condition, because of the curvature imposed by the stenotic pulmonary artery to the flow coming from the IVC, the competitive 


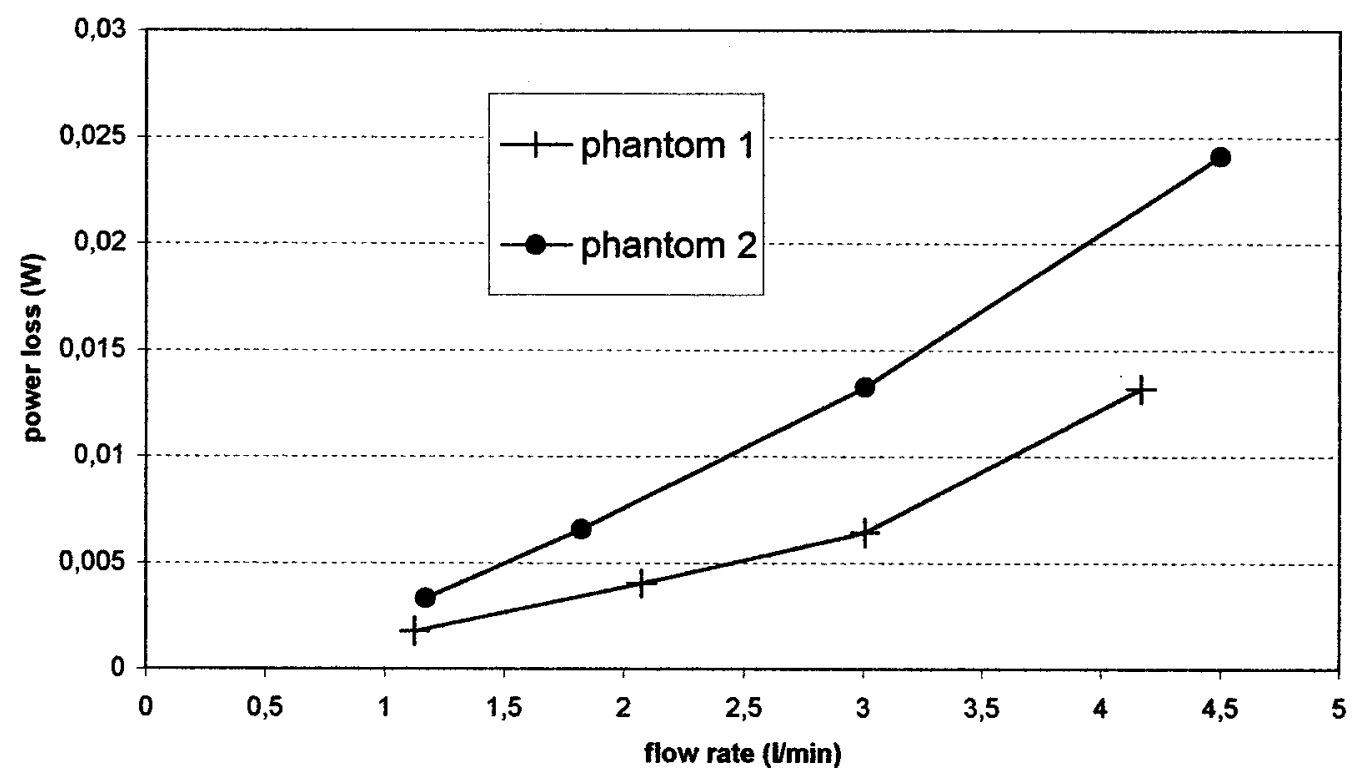

Figure 6. Power dissipation (see Equation 1) for both TECPC phantom circulations.

caval flows at the junction redirected predominantly toward the nonstenotic pulmonary artery, with large zones of positive vorticity (associated with counterclockwise flow rotation). The IVC flow was not streamlined, but appeared to split with the presence of zero velocity area at the center of a diverging zone.

In the type 2 phantom, with facing cavopulmonary anastomoses, the collision of the converging caval flows generated circular swirling patterns, secondary flows, and areas of stagnation characterized by low velocity (Figure 5). In comparison with the type 1 phantom, the flow was much less streamlined, and the flow exiting the venae cavae did not have a predominant direction toward either pulmonary artery. Flow visualization showed two vortices at the level of the center of the cross, where two swirling flows developed before entering into the pulmonary arteries.

\section{Power Dissipation}

The power loss, according to Equation 1, was analyzed relative to the two TECPC geometries considered, with the $60 \%$ of the total venous return carried by the IVC and the $40 \%$ by the SVC. The comparison of the dissipated power for the two different configurations confirmed the results of the flow visualization analysis: the type 2 phantom, which had a more disordered flow pattern, was found to have higher power losses than the configuration with an offset between venae cavae (Figure 6). It must be noticed that the type 1 phantom provided $1.4 \mathrm{~mW}$ at $1 \mathrm{~L} / \mathrm{min}$, whereas the type 2 phantom 2 under the same conditions had a value of $2.5 \mathrm{~mW}$, almost $80 \%$ higher. At 2 and $3 \mathrm{~L} / \mathrm{min}$ the power loss ratios of the type 2 phantom were $92 \%$ and $106 \%$, respectively, higher than those of the type 1 phantom.
At lower flow rates the difference was less pronounced, because the flow disturbances in the type 2 phantom were generated by slower caval flows and the kinetic energy at disposal was better managed by the connection than at higher flow rate. This result lends support to the hypothesis that this connection is more energetically efficient than the TECPC with zero offset. An interesting aspect of our measurements is that the power loss measurements did not follow a simple Poiseullian flow scheme. Actually, power loss values were higher than those obtainable with the simple calculation relative to a steady Poiseullian state. The reason for the higher dissipation may be found in the particular flow field generated by each caval connection topology (swirling, flow separation, vorticity transport, etc). This must be kept in mind in designing new Fontan geometries.

\section{Discussion}

Since the milestone work of de Leval and colleagues, ${ }^{2}$ an in vitro study that demonstrated the superiority of the TCPC with respect to the atriopulmonary connection, many institutions have achieved good early and midterm results with both intracardiac TCPC ${ }^{18}$ and TECPC. ${ }^{17}$ Recently several studies have considered the concept of energy conservation in the Fontan setup. Attention has focused on the best spatial arrangement of the systemic venous pathways. The demonstration of a better energetic efficiency related to the introduction of the offset between the caval inlets by in vitro ${ }^{7,8}$ and computational fluid dynamic studies ${ }^{15}$ has modified the surgical approach to the cavopulmonary connections.

The aim of our study was to determine the best TECPC geometry in terms of both energy preservation and safe clinical applicability. Since 1989, we have adopted TECPC 
as the principal technique for completion of Fontan operation. During these years we realized that when diverging cavopulmonary anastomoses are performed with the IVC inflow on the right of the bidirectional Glenn anastomosis, the IVC conduit may fall too close to or, nearly always, on the right lower lobar artery. This in turn may be deleterious for the following reasons. First, the right lower lobar artery extends in different plane and orientation than the IVC conduit-RPA anastomosis, and this may cause kinking of the artery itself, with impairment of right lung perfusion (in our experience one patient required takedown of the Fontan operation for acquired stenosis of the right lower lobe artery). Second, a relatively rigid structure, such as the prosthetic IVC conduit, may easily impinge on a thin vessel, such as a lobar artery. Third, the IVC connection to a zone of bifurcation and partition of flows is characterized by a worse hydrodynamic pattern than in the case of connection to a main vessel. In this respect, the clinical application of the elegant in vitro models of Sharma and associates ${ }^{7}$ and Enseley and colleagues ${ }^{8}$ with the IVC conduit placed on the right of the SVC results, in fact, in at least part of the conduit falling in the area of the first pulmonary bifurcation. We believe that it is more convenient to maintain a distance between the conduit and the distal pulmonary branchings to prevent potential impairment in lobar perfusion and obtain a better hydrodynamic pattern.

When we analyzed our results, the energetic advantage of the type 1 phantom relative to the type 2 phantom was evident. In fact, in the latter model collision and interaction of caval flows resulted in kinetic energy losses and viscous dissipation that decreased fluid dynamic efficiency. Our currently preferred pattern is therefore with the left-sided position of the extracardiac conduit, with some degree of offset and flaring. In this case the in vitro study showed that the inlet caval flows appeared to be directed away from each other, avoiding significant collision of the inlet flows with the presence of a vortex that seemed to play a beneficial role as a flow division structure.

As matter of fact, this apparent vortex was not a true vortex (a place of accumulation of vorticity). More correctly, it was a circulation zone, because such a rotation would not necessarily be associated with an accumulation of vorticity at its center. Moreover, the left-sided position of the conduit, in addition to offering a higher simplicity of offsetting and flaring, is potentially less compressive on the right pulmonary veins. A reason for concern with the leftsided position of the conduit could be that in older children the higher proportion of blood flow carried by the IVC is directed to a smaller and less compliant left lung. Salim and coworkers ${ }^{19}$ recently demonstrated that caval contributions to the total venous return vary with age in healthy children, from a low in the IVC of $45 \%$ at 2.2 years old to a high of $65 \%$, the adult value, at 6.6 years old. The distribution of the caval flow to each pulmonary artery is a function of the amount of blood that each lung can accommodate and theoretically depends on the amount of flow carried by each vena cava and on the geometry of the systemic venous pathway. Fogel and associates ${ }^{14}$ showed that in their patients with TCPC an equal amount of blood goes to both lungs even though the right lung is larger and could handle more blood. Tamir and colleagues ${ }^{20}$ and del Torso and associates $^{21}$ showed no evidence of predominant right lung perfusion in patients who had undergone atriopulmonary connection. None of these patients had a lateral tunnel that could influence the distribution of blood flow because of geometric orientation; instead, they had an atriopulmonary anastomosis, which theoretically could privilege the right lung perfusion. Therefore we can assume that curving the conduit preferentially toward one lung does not necessarily force a preferential or unbalanced flow split. Recent studies have pointed out the importance of the abnormality of the shear stress at the vessel anastomoses acting on vascular endothelium and altering cell function and structure, ${ }^{22}$ potentially modifying the release of vasoconstrictor agents, and ultimately altering the fate or behavior of the cavopulmonary hemodynamics. We believe that another advantage of the left-sided position of the IVC conduit could be a reduced shear stress at the anastomotic level.

In conclusion, the hemodynamic parameters and the biologic changes that affect the performance and the longevity of the cavopulmonary anastomoses in growing patients are still nebulous. On the basis of our clinical experience and this in vitro study, we suggest that the left-sided position of the IVC conduit with respect to the SVC is safer and permits an easier offsetting and flaring of the anastomosis. This arrangement avoids significant collision of the inlet flows, with formation of an apparent vortex, a weakly dissipative beneficial flow division structure, that ensures energy preservation.

\section{References}

1. Fontan F, Baudet E. Surgical repair of tricuspid atresia. Thorax. 1971;26:240-8.

2. de Leval MR, Kilner P, Gewilling M, Bull C, McGoon DC. Total cavopulmonary connection: a logical alternative for complex Fontan operation. J Thorac Cardiovasc Surg. 1988;96:682-95.

3. Puga FG, Chiavarelli M, Hagler DJ. Modifications of the Fontan operation applicable to patients with left atrioventricular valve atresia or single atrioventricular valve. Circulation. 1987;76(3 Pt 2):III53-60.

4. Marcelletti C, Corno A, Giannico S, Marino B. Inferior vena cavapulmonary artery extracardiac conduit : a new form of extracardiac bypass. J Thorac Cardiovasc Surg. 1990;100:228-32.

5. Laks H, Ardehali A, Grant PW, Permut L, Aharon A, Kuhn M, et al Modification of the Fontan operation: superior vena cava to left pulmonary artery connection and inferior vena cava to right pulmonary artery connection with adjustable atrial septal defect. Circulation. 1995;91:2943-7.

6. Kim YH, Walker PG, Fontaine AA, Panchal S, Ensley AE, Oshinski J, et al. Hemodynamics of the Fontan connection: an in-vitro study. J Biomech Eng. 1995;117:423-8. 
7. Sharma S, Goudy S, Walker P, Panchal S, Ensley A, Kanter K, et al. In vitro flow experiments for determination of optimal geometry of total cavopulmonary connection for surgical repair of children with functional single ventricle. J Am Coll Cardiol. 1996;27:1264-9.

8. Enseley AE, Lynch P, Chatzimavroudis P, Lucas C, Sharma S, Yoganathan A. Toward designing the optimal total cavopulmonary connection: an in vitro study. Ann Thorac Surg. 1999;68:1384-90.

9. Grigioni M, Amodeo A, Daniele C, D'Avenio G, Formigari R, Di Donato RM. Particle image velocimetry analysis of the flow field in the total cavopulmonary connection. Artif Organs. 2000;24:946-52.

10. Be'eri E, Maier SE, Landzberg J, Chung T, Geva T. In vivo evaluation of Fontan pathway flow dynamics by multidimensional phase-velocity magnetic resonance imaging. Circulation. 1998;98:2873-82.

11. Sharma S, Enseley AE, Hopkins K, Chatzimavroudis GP, Healy M, Tam VK, et al. In vivo flow dynamics of the total cavopulmonary connection from three dimensional multislice magnetic resonance imaging. Ann Thorac Surg. 2001;71:889-98.

12. Lardo AC, Webber SA, Friehs I, del Nido PJ, Cape GE. Fluid dynamic comparison of intra-atrial and extracardiac total cavopulmonary connections. J Thorac Cardiovasc Surg. 1996;117:697-704.

13. Fogel MA, Weinberg PM, Hoydu A, Hubbard A, Rychik J, Jacobs M, et al. The nature of flow in the systemic venous pathway measured by magnetic resonance blood tagging in patients having the Fontan operation. J Thorac Cardiovasc Surg. 1997;114:1032-41.

14. Fogel MA, Weinberg PM, Rychik J, Hubbard A, Jacobs M, Spray TL, et al. Caval contribution to flow in the branch pulmonary arteries of Fontan patients with a novel application of magnetic resonance presaturation pulse. Circulation. 1999;99:1215-21.

15. De Leval MR, Dubini G, Migliavacca F, Jalali H, Camporini G, Redington A, et al. Use of computational fluid dynamics in the design of surgical procedures: application to the study of competitive flows in cavopulmonary connections. J Thorac Cardiovasc Surg. 1996;111: 505-13.

16. Van Haesdonk JM, Mertens L, Sizaire R, Montas G, Purnode B, Daenen W, et al. Comparison by computerized numeric modeling of energy losses in different Fontan connections. Circulation. 1995;92(9 Suppl):II322-6.

17. Amodeo A, Galletti L, Marianeschi M, Picardo S, Giannico S, Di Rienzi P, et al. Extracardiac Fontan operation for complex cardiac anomalies: seven years' experience. J Thorac Cardiovasc Surg. 1997; 114:1020-30.

18. Stamm C, Friesh I, Mayer JE, Zurakoswki D, Triedman JK, Moran $\mathrm{AP}$, et al. Long-term results of the lateral tunnel Fontan operation. J Thorac Cardiovasc Surg. 2001;12:28-41.

19. Salim MA, DiSessa TG, Arheart KL, Alpert BS. Contribution of superior vena caval flow to total cardiac output in children: a Doppler echocardiographic study. Circulation. 1995;92:1860-5.

20. Tamir A, Melloul M, Berant M, Horev G, Lubin E, Blieden LC, et al. Lung perfusion scans in patients with congenital heart defects. J Am Coll Cardiol. 1992;19:383-8.

21. del Torso S, Milanesi O, Bui F, Benetti E, Stellin G, Mazzucco A, et al. Radionuclide evaluation of lung perfusion after the Fontan procedure. Int J Cardiol. 1988;20:107-16.

22. Morgan VL, Graham TP Jr, Lorenz CH. Alterations in pulmonary artery flow patterns and shear stress determined with three-dimensional phase-contrast magnetic resonance imaging in Fontan patients. J Thorac Cardiovasc Surg. 1998;116:294-304.

23. Grigioni M, Pedrizzetti G, Amodeo A, Daniele C, D'Avenio G, Zovatto L, et al. A comparison between numerical and PIV studies of the flow through a total cavopulmonary connection (abstract). Int $J$ Artif Organs. 2000;23:579.

\section{Appendix 1}

The 2-dimensional PIV technique used in this study is based on the cross-correlation of the intensity of two successive particle images, taken at an appropriate temporal interval $(\Delta t)$ between them (suggested by the flow properties). As shown in Appendix Figure 1, in a certain window of the original image the cluster of particles at a given instant will

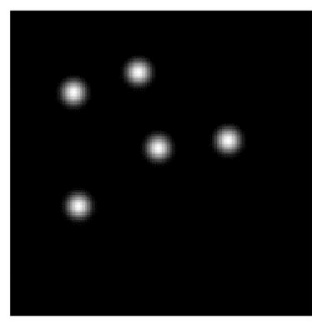

$\mathrm{t}$

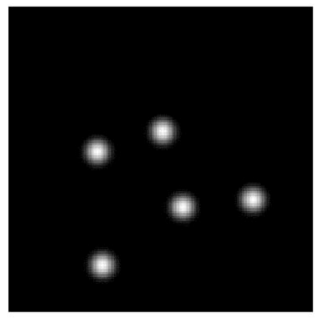

$t+\Delta \tau$
Appendix Figure 1. Pair of typical particle images analyzed with PIV technique. Interval $(\Delta t)$ between image recordings must be chosen to minimize loss of particles in second image while achieving appreciable displacement between images because of local velocity.

be subjected to a displacement, after $\Delta t$, related to the average local velocity of the flow. The highest peak in the correlation window of the PIV analysis, shown as a 3-dimensional graph in Appendix Figure 2, provides information about the mean displacement of particles during $\Delta \mathrm{t}$; thus the mean velocity in each particular location can be calculated by dividing this displacement by $\Delta \mathrm{t}$.

A total of 100 image pairs were used to calculate the velocity vector in each point of the flow field, yielding a high accuracy of the velocity measurements, both spatially and temporally. In fact, for a given ratio of the root-meansquare velocity field $u^{\prime}$ and the mean velocity $U$, the SD of the relative error in the determination of the mean velocity can be expressed as:

$$
\sigma=\frac{\mathrm{u}^{\prime}}{\sqrt{N \bar{U}}},
$$

where $N$ represents the number of velocity measurements used to calculate $u^{\prime}$ and $\bar{U}$. From this formula it can be calculated that the accuracy of our measurements was better than $2 \%$.

\section{Appendix 2}

The velocity components of a 3-dimensional flow field have some special properties in presence of a symmetry plane. In particular, only the two in-plane velocity components are different from zero and are even variables with respect to symmetry plane. The normal component is zero, but its normal derivative is different from zero. This nonzero derivative corresponds to flow entering or exiting the symmetry plane. Because of these facts the velocity on the symmetry plane is not divergence free, as it would be in a 2-dimensional approximation.

The velocity can be decomposed by the sum of an irrotational and a divergence-free component: 


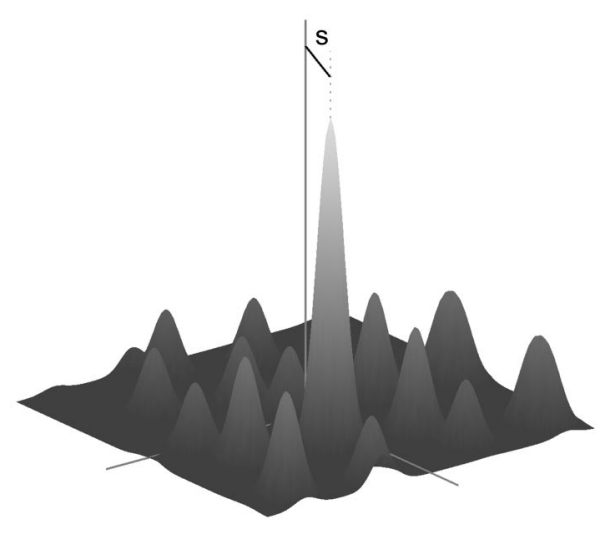

Appendix Figure 2. Cross-correlation function of intensity of images shown in Appendix Figure 1. Highest peak reveals average displacement $(s)$ of the particles during interval $(\Delta t)$.

$$
\begin{aligned}
& u_{x}=\frac{\partial \phi}{\partial x}-\frac{\partial \Psi}{\partial y}, \\
& u_{y}=\frac{\partial \phi}{\partial y}+\frac{\partial \Psi}{\partial x}
\end{aligned}
$$

where the stream function $\Psi$ and the potential $\phi$ satisfy the Laplace equations as follows:

$$
\begin{gathered}
\nabla^{2} \Psi=-\omega, \\
\nabla^{2} \phi=g .
\end{gathered}
$$

The variable $\psi(x, y)$ is the only nonzero vorticity component on the symmetry plane, and $g(x, y)$ is the divergence on the same plane. An equation for the vorticity can be written by expressing the Navier-Stokes equation in Taylor series near the symmetry plane. An equation for the flow divergence $g$ can also be obtained under the hypothesis that the symmetry plane flow is similar to the flow averaged on the fluid column flow above and below the symmetry plane. The system of partial differential equations is then solved by a finite-element formulation, which is second-order accurate in space and time. The derivation of the mathematic system and the numeric method of solution have been previously described elsewhere. ${ }^{23}$ 